\title{
Blinking chimeras in globally coupled rotators
}

Cite as: Chaos 29, 071101 (2019); https://doi.org/10.1063/1.5105367

Submitted: 27 April 2019 . Accepted: 10 June 2019. Published Online: 02 July 2019

Richard Janis Goldschmidt (D), Arkady Pikovsky (D), and Antonio Politi (i)

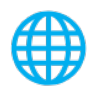

\section{ARTICLES YOU MAY BE INTERESTED IN}

Symmetry induced group consensus

Chaos: An Interdisciplinary Journal of Nonlinear Science 29, 073101 (2019); https:// doi.org/10.1063/1.5098335

\section{Solitary states for coupled oscillators with inertia}

Chaos: An Interdisciplinary Journal of Nonlinear Science 28, 011103 (2018); https:// doi.org/10.1063/1.5019792

Attractor reconstruction by machine learning

Chaos: An Interdisciplinary Journal of Nonlinear Science 28, 061104 (2018); https:// doi.org/10.1063/1.5039508

\section{AIP Author Services English Language Editing}




\title{
Blinking chimeras in globally coupled rotators
}

\author{
Cite as: Chaos 29, 071101 (2019); doi: 10.1063/1.5105367 \\ Submitted: 27 April 2019 . Accepted: 10 June 2019 \\ Published Online: 2 July 2019

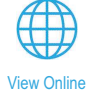 \\ Richard Janis Goldschmidt, ${ }^{1,2}$ (D) Arkady Pikovsky, ${ }^{1,3}$ (D) and Antonio Politi ${ }^{2}$ (D) \\ AFFILIATIONS \\ ${ }^{1}$ Department of Physics and Astronomy, University of Potsdam, Potsdam 10623, Germany \\ ${ }^{2}$ Institute of Pure and Applied Mathematics, University of Aberdeen, Aberdeen AB24 3FX, United Kingdom \\ ${ }^{3}$ Department of Control Theory, Lobachevsky University Nizhny Novgorod, Nizhny Novgorod 603022, Russia
}

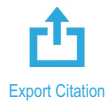

\begin{abstract}
In globally coupled ensembles of identical oscillators so-called chimera states can be observed. The chimera state is a symmetry-broken regime, where a subset of oscillators forms a cluster, a synchronized population, while the rest of the system remains a collection of nonsynchronized, scattered units. We describe here a blinking chimera regime in an ensemble of seven globally coupled rotators (Kuramoto oscillators with inertia). It is characterized by a death-birth process, where a long-term stable cluster of four oscillators suddenly dissolves and is very quickly reborn with a new reshuffled configuration. We identify three different kinds of rare blinking events and give a quantitative characterization by applying stability analysis to the long-lived chaotic state and to the short-lived regular regimes that arise when the cluster dissolves.
\end{abstract}

Published under license by AIP Publishing. https://doi.org/10.1063/1.5105367

Coupled oscillators can synchronize if the coupling is attractive or desynchronize if the coupling is repulsive. This basic effect is captured by the famous Kuramoto-Sakaguchi model of phase oscillators. However, if inertia is included, i.e., the units are rotators, more complex regimes in between synchrony and asynchrony can be observed. One such regime is a chimera pattern, where some rotators form a fully synchronous perfect cluster, while the others are nonsynchronized and all mutually different. In this paper, we report one such chimera characterized by an interesting additional long-time dynamics. We call it a "blinking chimera" because every once in a while an event occurs where the cluster opens up and quickly closes into a new reorganized composition. This event takes place on a time scale much shorter than that of the very long chaotic transient, that is, the chimera pattern-the system "blinks." We describe in detail how the exchange between the cluster and desynchronized units takes place.

\section{INTRODUCTION}

In the last few years, coupled oscillators proved to exhibit a very rich variety of regimes, ranging from perfect synchronization to extremely homogeneous asynchrony. The most intriguing regimes are the intermediate ones, especially when the oscillators spontaneously split into distinct groups/clusters. Among them, chimera states are currently attracting a large interest. They are characterized by the coexistence of synchronized and desynchronized groups of identical oscillators that, in spite of their indistinguishability, do not all behave in the same way. The first chimera was discovered by Kuramoto and Battogtokh ${ }^{1}$ in a one-dimensional medium of nonlocally coupled phase oscillators. Since then, many setups have been found, where symmetry is broken, giving rise to the simultaneous presence of synchronous and asynchronous subsets (see the reviews in Ref. 2 for both theoretical analyses and the description of experimental setups).

Furthermore, examples of chimera regimes within rather small sets of oscillators have been reported. ${ }^{3}$ Recent studies have revealed that chimera states can be quite complex. In particular, in breathing chimeras, ${ }^{4-7}$ some oscillators join and leave the synchronous domain because of oscillations of the governing order parameter.

In Secs. II-V, we report on a different steady nonstationary chimera. We describe a situation where a well-defined chimera persists for a very long time and is suddenly destroyed; shortly afterward, a new reshuffled and long-lived chimera reforms. Since the reshuffling events are rather short compared to the long chimera stages, we call this state "blinking chimera." Noteworthy, in the dynamics of networks, the notion of blinking systems is well established. ${ }^{8,9}$ There, switchings in the coupling and/or network topology are imposed according to a predefined external protocol-for example, periodic or random blinking. In our case, the blinking events are not predescribed, but appear spontaneously due to the dynamical rules.

The paper is organized as follows: we introduce the model in Sec. II and describe the phenomenology of blinking events in Sec. III. Next, we develop a quantitative characterization of blinking in Sec. IV. We conclude with a discussion in Sec. V. 


\section{THE BASIC MODEL}

The Kuramoto-Sakaguchi model (KS model from now) of globally coupled phase oscillators is a widely used system to study synchronization phenomena, for reviews, see Refs. 10-12. For identical units, the KS model is exactly solvable, ${ }^{12-14}$ yielding either complete synchronization (if the coupling is attractive) or an asynchronous state with vanishing order parameter (if the coupling is repulsive). The exact solution shows that chimera states, characterized by the coexistence of fully synchronous (identical) oscillators with asynchronous units, cannot arise.

Integrability breaks up if the original model is perturbed. One popular extension of the KS model consists in including the effects of inertia on the oscillating units, i.e., in replacing phase oscillators with rotators, ${ }^{15-23}$

$$
\alpha \ddot{\phi}_{i}+\dot{\phi}_{i}=\frac{1}{N} \sum_{j=1}^{N} \sin \left(\phi_{j}-\phi_{i}-\beta\right) .
$$

Here, $\phi_{i}$ is the instantaneous phase of the $i$-th rotator, $N$ is their number, $\beta$ is a constant phase shift (sometimes called "frustration" in the literature), and, finally, $\alpha$ is the (dimensionless) mass of the rotators. A constant torque acts on all rotators and the equations are written in the reference frame rotating with the corresponding constant frequency (thus, the torque does not enter).

At this point, it is instructive to discuss an essential difference between oscillators and rotators. For example, pendula can perform both oscillations and rotations. When they are used in pendulum clocks and in metronoms, ${ }^{24,25}$ they operate as self-sustained oscillators. For these oscillations, a phase can be introduced, which is different from the angle variable $\phi$ in (1) and obeys a first-order (in time) equation. Thus, for coupled oscillators (and for all setups of coupled metronoms), the usual first-order Kuramoto model is appropriate; the model "with inertia" cannot be used for oscillators, but for rotators only.

Systems of coupled rotators have been widely discussed in the literature. In Refs. 15 and 26, diversity of torques has been shown to result in a hysteretic transition to synchrony. Effects of noise and diversity have been treated analytically and semianalytically in Refs. 27-29. One of the popular applications of the rotator model of type (1) is power grids. ${ }^{17-19,26,30,31}$ In these applications, one does not consider a mean field coupling like in Eq. (1), but a network of different producers and consumers of electrical power, with different values of torque and different connectivities. Another much studied setup is that of symmetric deterministic models where chimera states emerge as a result of symmetry breaking. This is the case of a onedimensional medium with nonlocal coupling studied in Ref. 32 and of two symmetric subpopulations with different couplings (within and between them) considered in Ref. 33. Here, we consider a setup that is even "more" symmetric, since all pairwise interactions are equal to one another. The phenomena we thus observe are entirely due to the prescribed dynamics and cannot be attributed to diversity among the oscillators, network topology, or noise.

For $\alpha \rightarrow 0$, system (1) reduces to the standard KS system, describing the behavior of (identical) phase oscillators. Therefore, for small $\alpha$-values, the dynamics is expected to closely reproduce that of the KS model. In the limit $\alpha \rightarrow \infty$, the system converges to the
Hamiltonian mean field model: ${ }^{11}$ a paradigmatic model for the study of long-range interactions in the presence of a conservative dynamics.

We expect interesting and potentially new phenomena to arise in the region where attraction and repulsion nearly balance each other. This is indeed the parameter region where standard chimera states are observed. More specifically, we have selected $\beta=0.53 \cdot \pi$, which corresponds to a weakly repulsive coupling in the KS model, while in the KS model with inertia neither the fully synchronous nor the splay states are stable. Additionally, in order to investigate the role of inertia, we have selected a finite and relatively large mass $\alpha=10$. As for the number of oscillators, we assume $N=7$ : it is the smallest system size for which blinking chimeras have been observed. For $N<7$, we have observed only either simple clustered or fully disordered states.

\section{PHENOMENOLOGY}

In this section, we qualitatively describe chimera states and their blinking; a quantitative characterization and a more thorough analysis are postponed to Sec. IV.

The equations of motion (1) have been simulated by implementing a standard 4 th order Runge-Kutta integrator with a time step of $\Delta t=0.01$. Phases $\phi_{i}$ and frequencies $\dot{\phi}_{i}$ are initialized by drawing them from random distributions, $\phi_{i} \in \mathcal{U}(0,2 \pi)$ and $\dot{\phi}_{i} \in$ $\mathcal{N}(0,1)$. Moreover, we have introduced numerical inhomogeneities on the mass $\alpha$ of the order $\Delta \alpha \simeq O\left(10^{-14}\right)$ to prevent the oscillators "clumping together" due to finite floating point precision. We classify the current configurations by identifying clusters of oscillators in almost identical states. Two oscillators indexed by $i$ and $j$ are said to belong to the same cluster when their distance $d(i, j)<10^{-10}$, where

$$
d(i, j)=\sqrt{\delta\left(\phi_{i}-\phi_{j}\right)^{2}+\left(\dot{\phi}_{i}-\dot{\phi}_{j}\right)^{2}}
$$

and $\delta=\min \left(\left|\phi_{i}-\phi_{j}\right|, 2 \pi-\left|\phi_{i}-\phi_{j}\right|\right)$ to take into account that $\phi_{i}$ is equivalent to $\phi \pm 2 \pi$.

\section{A. Instability of the splay and the fully synchronous states}

Before describing the formation of the chimera state in detail, we comment on the instability of the completely synchronous and the fully-asynchronous (splay) solutions. The completely synchronous state is given by $\phi_{i}=\phi_{j}$ and $\dot{\phi}_{i}=\dot{\phi}_{j}, \forall i, j$, where all oscillators collapse into a cluster. We study the stability of this cluster via the transversal Lyapunov exponent [which tells us whether a virtual pair of oscillators would be repelled or attracted by the cluster, see Sec. IV and Eq. (4) below for a discussion]. The resulting second-order equation for the transversal perturbation $\alpha \ddot{\delta}+\dot{\delta}+$ $\cos \beta \delta$ can be solved analytically. The exponents are $\lambda_{1,2}=(-1 \pm$ $\sqrt{1-4 \alpha \cos \beta}) /(2 \alpha)$, yielding for the parameters under consideration $\lambda_{1} \approx 0.047$ and $\lambda_{2} \approx-0.147$. Hence, the fully synchronous cluster is unstable with transversal Lyapunov exponent $\lambda \approx 0.047$.

The splay state is characterized by the frequencies $\dot{\phi}_{i}=0, \forall i$, and an equidistribution of phases on the unit circle, $\phi_{i}=i$. $2 \pi / N, i \in\{0, N-1\}, N=7$ being the number of oscillators in the system. It is difficult to analyze the instability of this steady state analytically, but numerical exploration is straightforward: one easily 

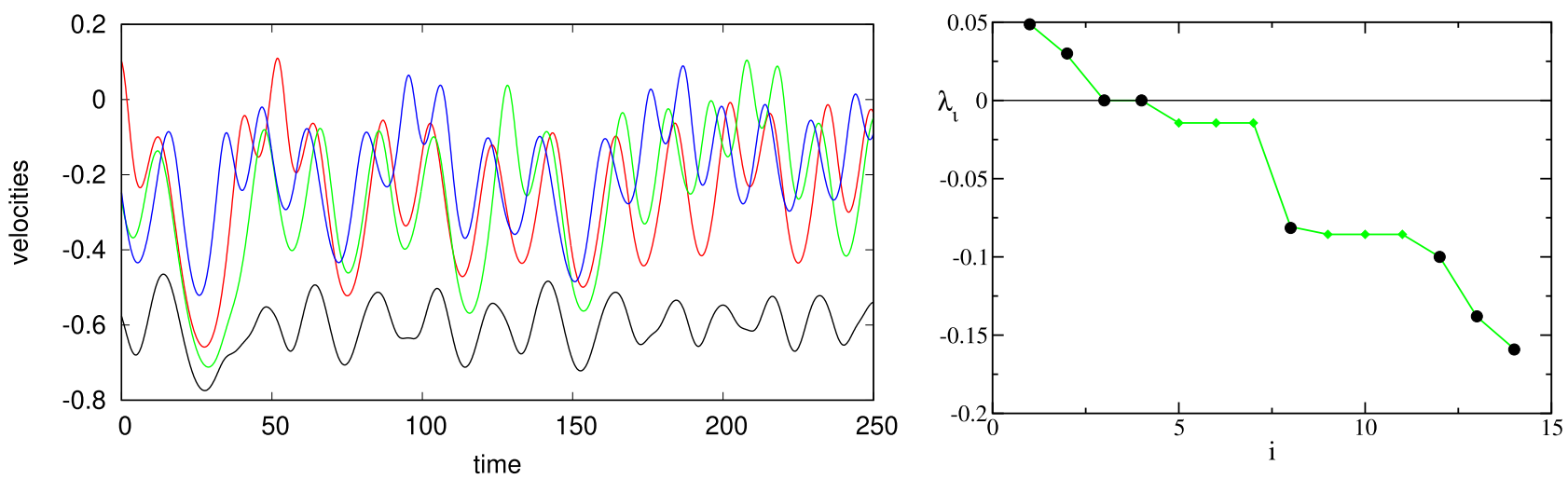

FIG. 1. Left panel: time series of rotator velocities in the chaotic chimera state. Red, blue, and green curves: isolated (not belonging to a cluster) units, black: cluster of 4 units. Right panel: the full Lyapunov spectrum in the chaotic regime (green symbols, partially overlapped with black ones). It has two positive Lyapunov exponents (thus, this regime can be characterized as hyperchaos), two zero LEs (due to two invariances-with respect to shift of time and with respect to shift of all phases), with all other exponents being negative. One can see a degeneracy due to the presence of a cluster: there are two groups of three equal LEs $(i=5,6,7$ and $i=9,10,11)$, which correspond to the transversal directions of the cluster, see discussion of the transversal LEs below. Other exponents (black circles) coincide with those of the reduced system (3), where the cluster configuration $4-1-1-1$ is fixed so that the system is 8 -dimensional and has 8 LEs.

observes that a small (of order $10^{-12}$ ) perturbation grows exponentially with the exponent $\lambda \approx 0.1$. Thus, both the fully synchronous cluster and the splay state are unstable.

\section{B. Formation of a chimera state}

The free evolution from random initial conditions leads to the formation of a chimera state, where four oscillators clump together to form a cluster, while the three other oscillators remain isolated from one another (we indeed refer to them as to "isolated units"). We denote this chimera state as 4-1-1-1. It appears that this state is a global attractor, as in our numerical simulations, we never observed other configurations formed from random initial conditions. Chimera states of this type have been observed in globally coupled identical phase oscillators with delay and in globally coupled Stuart-Landau oscillators. ${ }^{34-36}$ The average time for the formation of a chimera is $\approx 1.8 \cdot 10^{3}$. The corresponding dynamics is chaotic, as qualitatively visible in the left panel of Fig. 1, where we plot the time series of the rotator velocities $\dot{\phi}_{i}$. Here, all units are chaotic: those belonging to the cluster and the isolated ones. On a more quantitative level, the corresponding Lyapunov spectrum is plotted in the right panel. It is composed of 14 exponents, two of which are indeed larger than zero, two vanish (due to invariance under time translation and under a homogeneous shift of the phases), while all the others are negative.

In the spectrum, we also notice two triples of identical negative exponents. As confirmed below (see Sec. IV), they account for the transversal stability of the 4-cluster. The remaining eight Lyapunov exponents (see the filled black circles in Fig. 1) contribute to the dimension of the underlying attractor. By virtue of the Kaplan-Yorke formula, $D_{K Y} \approx 4.8$ represents an upper bound to the information dimension.

No other configurations have been observed in the system (1) for the same parameter values-neither fully synchronous states nor chimera-type configurations with 2,3,5, or 6 elements in the cluster.

\section{Blinking of chimera}

The 4-1-1-1 regime described above is observed on a relatively long-time scale, but it is not the asymptotic one. Over very long-time scales, one observes a picture like in Fig. 2: rare events lead to a reshuffiling of the cluster composition, with some oscillators leaving the cluster and others joining. These reshufflings are observed continuously, and they are apparently independent random events following a Poisson process with a rate $\approx 3.6 \cdot 10^{-7}$ (corresponding to a mean time $\approx 2.8 \cdot 10^{6}$ between the events). This follows from

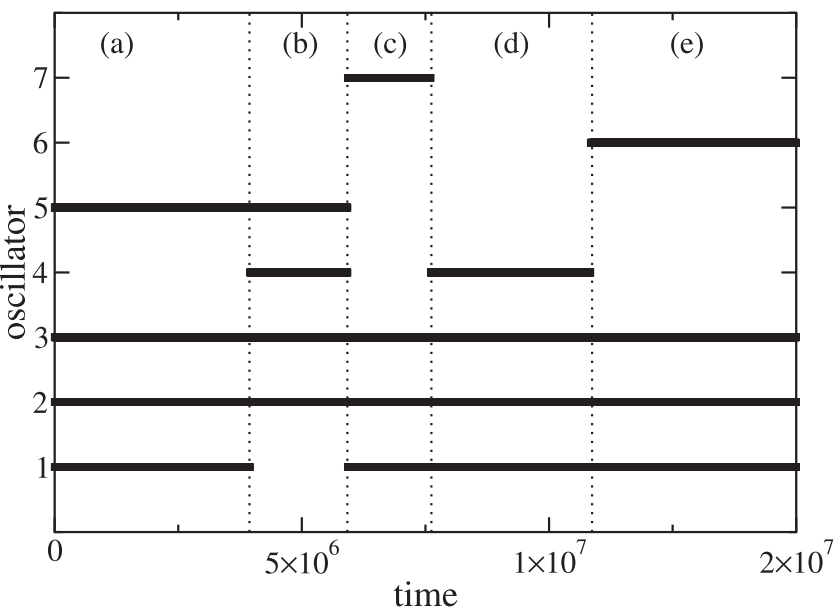

FIG. 2. Pattern of the oscillators belonging to the four-oscillator cluster (marked by black squares which are seen as bold lines) vs time. One can clearly see five epochs with different cluster compositions. In epoch (a), oscillators 1, 2, 3, and 5 belong to the four-cluster. In epoch (b), oscillators 2, 3, 4, and 5; in (c), oscillators $1,2,3$, and 7 ; in (d), oscillators 1, 2, 3, and 4; finally, in epoch (e), oscillators 1, 2, 3 , and 6 belong to the cluster. 


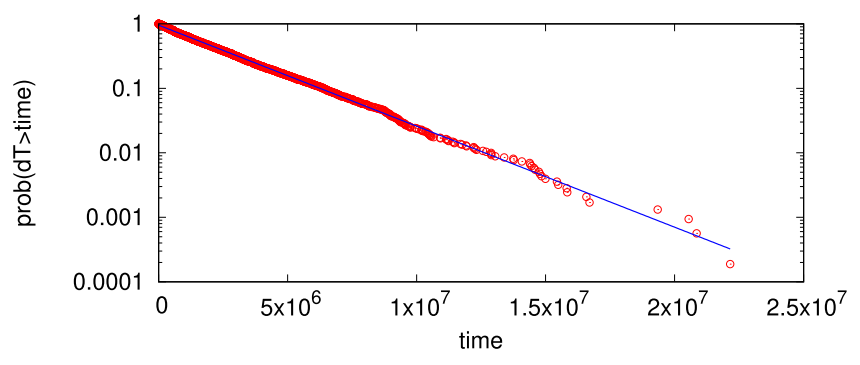

FIG. 3. Distribution of time intervals between different reshuffling events.

an exponential distribution of interevent time intervals, presented in Fig. 3.

The observed pattern of reshuffling events shows that the chimera state is not stationary, but blinking. Below we provide further illustrations of this blinking and characterize it more quantitatively.

\section{Destruction and reformation of a 4-1-1-1 chimera state}

Here, we describe in detail, mostly qualitatively, what happens during the blinking (reshuffling) events. In fact, we have found that three different reshuffling scenarios can happen; they are presented in Figs. 4-6. We refer to these cases as to A, B, and C. We first describe scenario A with reference to Fig. 4; it will then be straightforward to explain also the other two scenarios.

In the two panels of Fig. 4, we show distances between oscillators, defined according to Eq. (2), as a function of time (time offset is chosen arbitrarily at some instant around 1000 units prior the start of reshuffling). In this event, the initial 4-cluster configuration contains the units 4, 5, 6, and 7, while the reshuffled 4-cluster contains the units $2,4,6$, and 7 . Accordingly, in the top panel, we depict distances from unit 4 , which belongs to the cluster both prior and after reshuffling (in the same way, we always choose the reference unit as belonging to the cluster prior and after the event in top panels of Figs. 5 and 6). In the bottom panel, we show distances between all the three pairs of units not belonging to the cluster prior to reshuffling.

In the top panel of Fig. 4, one can appreciate the presence of a chaotic $4-1-1-1$ state for $t \lesssim 800$. It is followed by a regular regime during which the isolated unit 2 comes rather close to the 4 -cluster. In this regime, the 4-cluster is unstable and starts dissolving. Meanwhile, the units 1 and 3 come close to each other. The dissolution of the 4-cluster, accompanied by the appearance of a 2-cluster emerging from the isolated units, continues until $t \approx 2000$. Afterward, the 2 -cluster dissolves and the dynamics again become chaotic. Around $t \approx 3000$, no clusters are observed. Four units are close to each other, although they do not fulfill our criterion for the definition of a cluster. However, they start approaching each other and a new 4-cluster eventually forms in the same way as observed when starting from random initial conditions (cf. Sec. III B above). Typically, the unit which was already close to the 4 -cluster around $t \approx 1000$, joins the novel cluster, "exchanging" with one unit that leaves the cluster. However, in some cases the temporary 2-cluster does not dissolve but enters the novel 4-cluster, exchanging with two units therein.
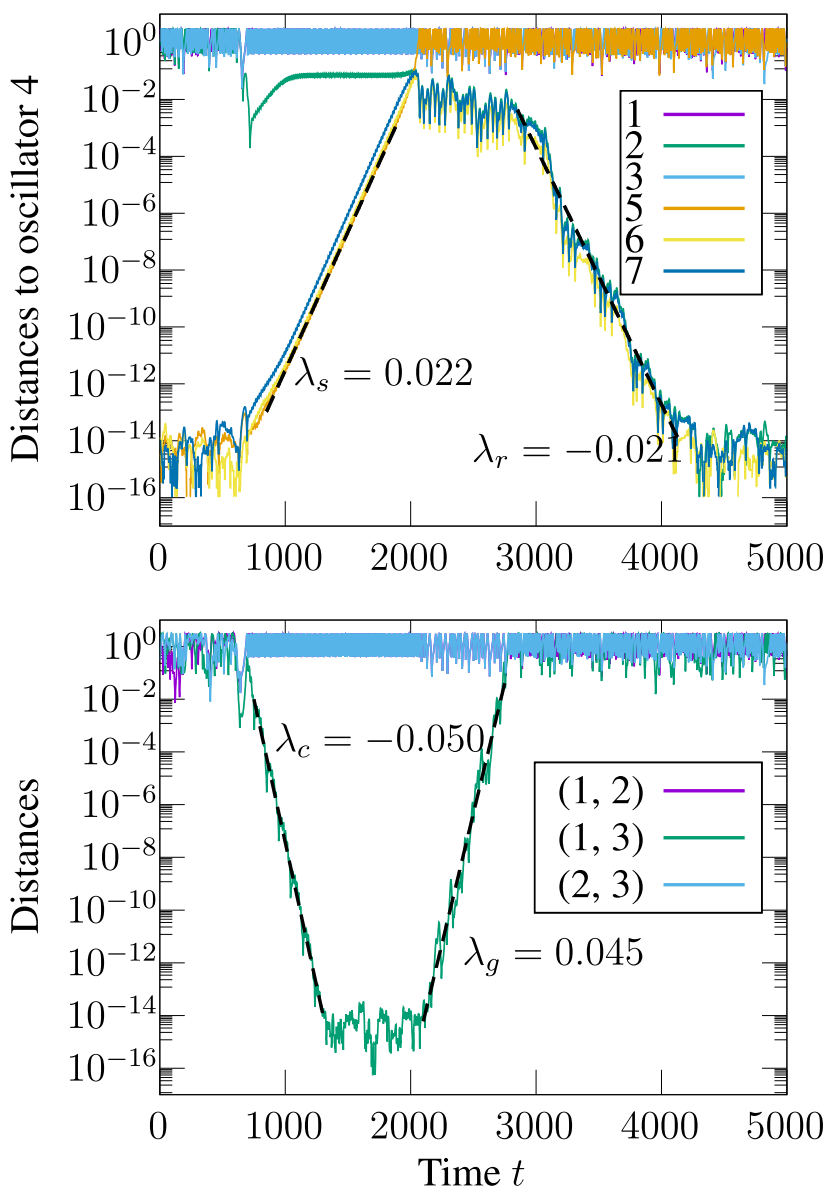

FIG. 4. Reshuffling of a 4-1-1-1 chimera state: scenario A. Top panel: distances of all oscillators from oscillator 4 . Isolated oscillators at the beginning are 1 , 2,3 , distances between them are depicted in the bottom panel. Around $t \approx 700$, a regular regime emerges from the chaotic 4-1-1-1 state, with one isolated oscillator (here unit 2). Simultaneously, the 4-cluster begins to disintegrate (with a rate $\lambda_{s}$ ) and two of the initially isolated units come close and form a 2-cluster state (here units 1,3 ). (bottom panel, rate $\lambda_{c}$ ). Disintegration ends at $t \approx 2000$ in a chaotic state, where the units $2,4,6,7$ form a not so perfect 4-cluster (mutual distances are $\approx 10^{-2}$ ), while the units 1, 3 form a 2-cluster (see bottom panel), and unit 5 is isolated. The 2-cluster begins to disintegrate with a rate $\lambda_{g}$; this disintegration ends around $t \approx 2800$. From this moment on, the new 4-cluster becomes more stable, the mutual distances reduce with the rate $\lambda_{r}$. At the end of the event, at $t \approx 4000$, a reshuffled configuration $4-1-1-1$ appears.

The blinking event shown in Fig. 5 is quite similar to that of Fig. 4, with the following differences: (i) now, during the regular state arising at the beginning of reshuffling (around $t \approx 1000$ ), not one, but two isolated units "orbit" close to the 4-cluster (here units 2 and 5); (ii) the disintegration of the 4-cluster is much faster than in Fig. 4; (iii) the two isolated units that were close to the 4 -cluster join the new cluster, so there is always a $2 \leftrightarrow 2$ exchange.

The blinking event in Fig. 6 is different from those in Figs. 4 and 5: here there is no formation of a temporary 2-cluster. The breakup of the 4 -cluster (at $1000 \lesssim t \lesssim 1600$ ) is faster than in case 

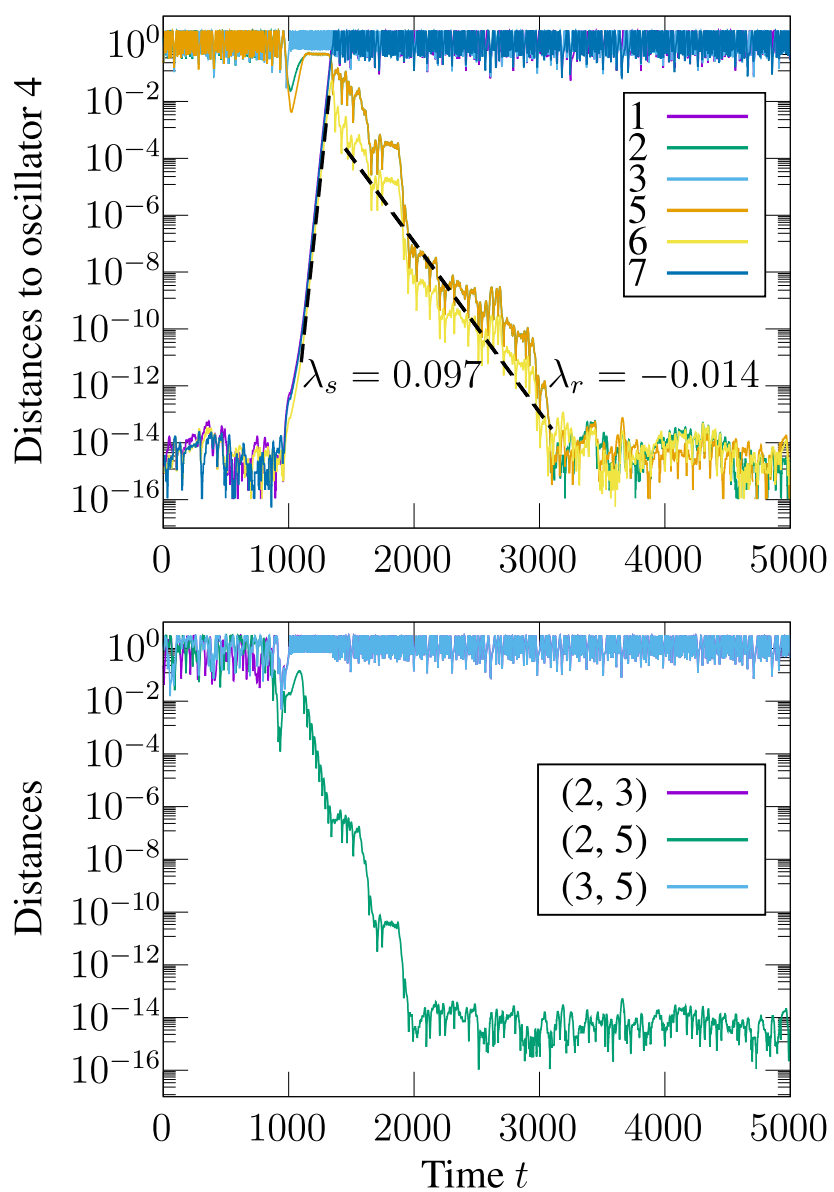

FIG. 5. Reshuffling of a 4-1-1-1 chimera state: scenario B. Top panel: distances of all oscillators from oscillator 4 . Isolated oscillators at the beginning are $2,3,5$; distances between them are depicted in the bottom panel. The process is qualitatively similar to that of $\mathrm{Fig}$. 4, but here in the regular stage around $t \approx 1000$, two isolated oscillators $(2$ and 5$)$ are both close to each other and to the still existing cluster; they eventually join the new 4-cluster.

Fig. 4, but slower than in case Fig. 5. At the end of this process, all the units are separated, and a new 4 -cluster begins to form, with a $2 \leftrightarrow 2$ exchange. Sometimes, we observed that all 3 previously isolated units join the new cluster so that also a $3 \leftrightarrow 3$ exchange is possible. On the other hand, since the formation of a new cluster is a statistical process, it can happen that the final configuration is formed of the same units as the initial one.

\section{QUANTIFYING BLINKING EVENTS}

The above description of the reshuffling process suggests the existence of regular (nonchaotic) temporary stages. To resolve them, we proceed by performing simulations with four units "glued" together to enforce the presence of a 4-cluster at all times. Such a state (and, more generally, any clustered state) can be modeled also with "reduced" equations, the identical elements of a cluster being described by just one set of variables $(\varphi, \dot{\varphi})$. Generally, if $N$ rotators
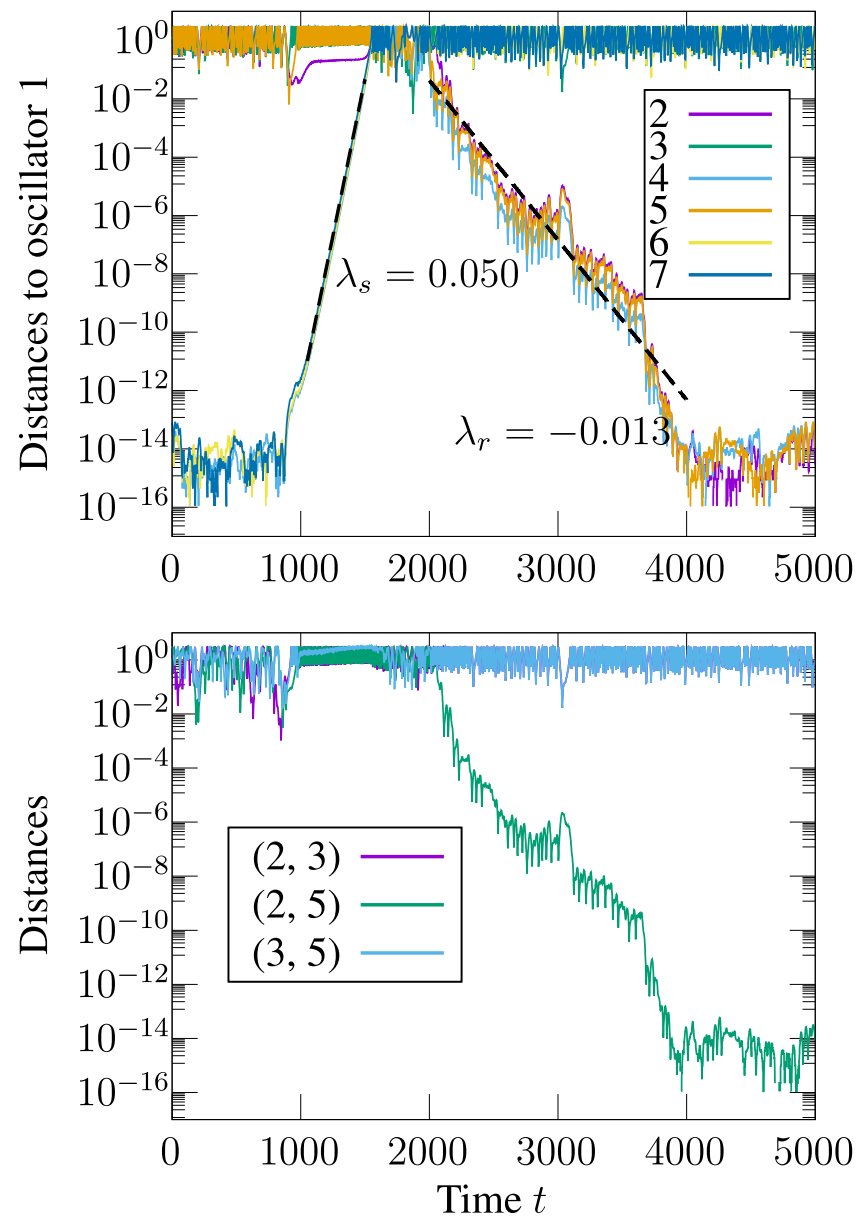

FIG. 6. Reshuffling of a 4-1-1-1 chimera state: scenario C. Top panel: distances of all oscillators from oscillator 1 . Isolated oscillators at the beginning are $2,3,5$; distances between them are depicted in the bottom panel. At the end units $1,2,4,5$ belong to the 4 -cluster and units $3,6,7$ are isolated.

build $K$ clusters of sizes $n_{1}, n_{2}, \ldots, n_{K}$, with $\sum_{k} n_{k}=N$, then Eq. (1) can be rewritten as a smaller reduced system of $K$ equations,

$$
\alpha \ddot{\varphi}_{k}+\dot{\varphi}_{k}=\operatorname{Im}\left(Z \mathrm{e}^{-i \varphi_{k}-i \beta}\right), \quad Z=\sum_{k=1}^{K} \frac{n_{k}}{N} \mathrm{e}^{i \varphi_{k}} .
$$

In our case of a 4-1-1-1 chimera, we have $K=4$ with $n_{1}=4, n_{2}=n_{3}=n_{4}=1$. The reduced system (3) has the same dynamics as the original system (1) so long as the 4-cluster persists.

In fact, simulations of the 4-1-1-1 chimera state with Eq. (3) reveal that this regime is nothing but a very long pseudostationary chaotic transient. ${ }^{37}$ The dynamics, eventually, collapses onto one of the following four attractors:

A: a quasiperiodic 4-2-1 state, characterized by a 2-cluster, while the isolated oscillator orbits close to the 4-cluster;

B: a quasiperiodic $4-2-1$ state such that the 2-cluster orbits close to the 4-cluster; 
C: a periodic 4-1-1-1 state, where three units remain asymptotically isolated; and

D: a periodic $4-3$ state.

Upon performing 1000 simulations of the reduced model, starting from random initial conditions, we found that roughly $54 \%, 31 \%$, $13 \%$, and $2 \%$ converge to attractor $\mathrm{A}, \mathrm{C}, \mathrm{B}$, and $\mathrm{D}$, respectively. We have used the notations $\mathrm{A}, \mathrm{B}, \mathrm{C}$, because these regular states correspond to the scenarios A, B, and C discussed in Sec. III D. As for the probability to observe the various scenarios in the original blinking dynamics, they are approximately equal to the above reported rates, the major difference being that the state $\mathrm{D}$ has been never observed in the original system (1), presumably because the 4-cluster is destroyed prior to the formation of the 3-cluster.

The reason why the attractors of the reduced model are not seen as such in the simulations of the global system is that the 4-cluster is transversally unstable in all of the above scenarios. Transversal Lyapunov exponents can be determined by perturbing only the variables of the cluster $\left(\varphi_{k}, \dot{\varphi}_{k}\right)$, while leaving the mean field $Z$ unchanged (cf. the general discussion of transversal Lyapunov exponents in Ref. 38). So the linear equation for a deviation $\left(\delta_{k}, \dot{\delta}_{k}\right)$ from cluster $k$ reads

$$
\alpha \ddot{\delta}_{k}+\dot{\delta}_{k}=-\delta_{k} \operatorname{Re}\left[Z e^{-i \beta-i \varphi_{k}}\right] .
$$

In practice, the above equation, solved together with Eq. (3), tells us whether a virtual pair of oscillators leaving cluster $k$ would be either attracted or repelled by the cluster. As Eq. (4) is twodimensional, it yields two transversal Lyapunov exponents; we are interested in the maximal one that can be computed in the usual way by virtue of the Benettin algorithm, ${ }^{38}$ i.e., by regularly renormalizing the vector $\left(\delta_{k}, \dot{\delta}_{k}\right)$ and averaging the logarithm of the norm.

The implementation of this approach during the pseudostationary transient evolution of the 4-1-1-1 configuration yields two values that coincide with the two triples visible in the general Lyapunov spectrum plotted in Fig. 1 and confirm that the 4-cluster is stable on average. In the case of the above mentioned four attractors, we instead find that the largest transversal Lyapunov exponent is $\lambda_{t}^{A} \approx 0.0227$ [for case A]; $\lambda_{t}^{B} \approx 0.1\left[\mathrm{~B}\right.$ ]; $\lambda_{t}^{C} \approx 0.05[\mathrm{C}] ; \lambda_{t}^{D} \approx 0.17$ [D], thus confirming the instability of the 4-cluster.

Regular attractors do not only qualitatively correspond to the initial states of the blinking events described in Sec. III D, but also give a quantitative description of the disintegration of the 4-cluster: the growth rates of the interoscillator distances in Figs. 4-6 correspond to the values of the transversal Lyapunov exponents for cases A-C.

Additional insight into cases A and B can be obtained from the computation of the transversal Lyapunov exponents of the 2-cluster in the corresponding 4-2-1 configuration. In both cases, its value is $\approx-0.05$. This quantity describes the rate $\lambda_{c}$ with which 2 -cluster is formed, cf. Figs. 4 and 5.

The formation of a new 4-cluster from a nonclustered chaotic regime is a statistical event. However, its late stage, where the cluster is basically formed and the units are progressively approaching each other, can be again compared with the transversal Lyapunov exponents. In this context, the stable transversal exponent $\lambda_{t} \approx-0.014$ of the 4-cluster in the chaotic regime (Fig. 1) is relevant, as it gives approximately the convergence rate $\lambda_{r}$ in Figs. 4-6.
Finally, the rate of disintegration of the two-cluster $\lambda_{g}$ (bottom panel of Fig. 4) can be explained as follows: this regime corresponds to a temporary chaotic state in the fixed configuration 4-2-1. Calculations of the transversal Lyapunov exponent of the 2-cluster here are not reliable, as this regime ends quite soon in one of the A, B, D states. However, if one starts the configuration 4-2-1 from random initial conditions, in many runs, one observes that during the initial stages the transversal Lyapunov exponent of cluster 4 fluctuates around zero, while the transversal LE of cluster 2 is $\approx 0.048$. This value corresponds to the rate $\lambda_{g}$ of disintegration of the 2 -cluster in Fig. 4.

Summarizing, we explained the origin of blinking events via an interplay of two properties of the system: structural, i.e., the composition of clusters, and dynamical, i.e., the complexity of the dynamics and the resulting stability characteristics of clusters. During long epochs a chaotic regime with stable 4-1-1-1 clustering is observed. However, after a long but finite time, chaos is succeeded by a regular regime, and this triggers a blinking event: first, the big cluster becomes unstable and dissolves, and then from a chaotic disordered state, a new chimera state with a reshuffled composition emerges.

\section{DISCUSSION AND CONCLUSIONS}

In this paper, we reported on a novel state of blinking chimera in a small system of seven identical rotators (phase oscillators with inertia). The asymptotically stationary regime consists of a sequence of long epochs each characterized by a (temporary) chaotic chimera state with four oscillators synchronized into a single cluster and three isolated ones. Such regimes are separated by relatively short reshuffling events when the composition of the synchronous cluster is reconfigured. These rare events, which we call "blinking events," appear to be distributed according to a Poisson process with a very small, but finite rate. There are three types of such events (see the above described scenarios A, B, and C); all of them are characterized by a regular (either periodic or quasiperiodic-depending on the scenario) dynamics.

Altogether, the chaotic chimera state is not an attractor, but rather a transient chaotic state, eventually ending in a temporary regular dynamics. The emergence of long chaotic transients is a well known phenomenon in nonlinear dynamics: ${ }^{37}$ they typically arise because of "holes" in phase space, where the trajectory suddenly jumps out of the pseudoattractor. Identifying the specific conditions for these events to occur is not an easy task: we leave it to future investigations. What makes the regime discussed in this paper different from standard chaotic transients is that once the temporary chaotic state is over, another equivalent such regime emerges. In fact, the three types of exit events all lead to unstable attractors. In practice, during the blinking event, the cluster is transversally unstable and it thereby starts disintegrating, leading to a short-lived nonchimera stage. A new chimera configuration of the type 4-1-1-1 "finally" forms, due to the transversal stability of this regime.

In order to clarify the quantitative properties of these processes, we explored the dynamics of restricted systems with fixed cluster compositions. This allowed us to calculate, via time averaging, the transversal Lyapunov exponents governing the stability of the clusters, without destroying the clusters themselves. The fixed configuration 4-1-1-1 allowed us to determine the basic unstable transversal 
Lyapunov exponents governing disintegration of the main 4-cluster. In some cases, an intermediate 2-cluster is formed, with this formation being governed by the stable Lyapunov exponent of the 2-cluster in the fixed 4-2-1 configuration. Finally, the rate of formation of the new 4-cluster from the unclustered chaotic state is governed by the stable transversal Lyapunov exponent of the 4-cluster in the chaotic transient state of the 4-1-1-1 configuration.

From the general viewpoint of topology of the dynamics in the phase space of the system, the blinking chimera can be described as follows: there is an invariant manifold where the states of four oscillators coincide, while three differ. This 8-dimensional manifold is attractive for a set of large measures in the original 14-dimensional phase space but is not a global attractor. On this invariant set, a chaotic transient (chaotic saddle) sets in, characterized by a very long lifetime: this regime corresponds to a chaotic chimera. Eventually, generic trajectories leave the chaotic saddle and approach one of the sets $(\mathrm{A}, \mathrm{B}, \mathrm{C})$ all characterized by a regular dynamics. On these regular sets, the 8-dimensional manifold is transversally unstable so that trajectories leave it (the chaotic chimera is destroyed), but then enter again the domain of attraction of the chaotic 8dimensional saddle, and a new, reshuffled chimera is established. Noteworthy, intermittent chaotic chimeras have been reported for a two-population setup. ${ }^{33}$ However, no reshuffling, and thus also no blinking, was observed therein.

Preliminary simulations suggest that this phenomenon is not peculiar of the parameters selected in this paper. However, in our simulations, we never observed blinking chimera for less than seven units. Therefore, in this short communication, we restricted ourselves to a description of the minimal blinking chimera and do not discuss other possible dynamical states of this system.

\section{ACKNOWLEDGMENTS}

We thank M. Rosenblum and Yu. Maistrenko for useful discussions. This work has been funded by the EU's Horizon 2020 Research and Innovation Programme under the Marie Sklodowska Curie Grant Agreement No. 642563. A. Pikovsky acknowledges support of the Russian Science Foundation (Grant No. 17-12-01534).

\section{REFERENCES}

${ }^{1}$ Y. Kuramoto and D. Battogtokh, "Coexistence of coherence and incoherence in nonlocally coupled phase oscillators," Nonlinear Phenom. Complex Syst. 5, 380-385 (2002).

${ }^{2}$ M. J. Panaggio and D. M. Abrams, "Chimera states: Coexistence of coherence and incoherence in networks of coupled oscillators," Nonlinearity 28, R67-R87 (2015).

${ }^{3}$ Y. Maistrenko, S. Brezetsky, P. Jaros, R. Levchenko, and T. Kapitaniak, "Smallest chimera states," Phys. Rev. E 95, 010203 (2017).

${ }^{4}$ F. P. Kemeth, S. W. Haugland, L. Schmidt, I. G. Kevrekidis, and K. Krischer, "A classification scheme for chimera states," Chaos 26, 094815 (2016).

${ }^{5}$ M. I. Bolotov, L. A. Smirnov, G. V. Osipov, and A. S. Pikovsky, "Breathing chimera in a system of phase oscillators," JETP Lett. 106, 393-399 (2017).

${ }^{6} \mathrm{M}$. Bolotov, L. Smirnov, G. Osipov, and A. Pikovsky, "Simple and complex chimera states in a nonlinearly coupled oscillatory medium," Chaos 28, 045101 (2018).

${ }^{7}$ Y. Suda and K. Okuda, "Breathing multichimera states in nonlocally coupled phase oscillators," Phys. Rev. E 97, 042212 (2018).

${ }^{8}$ I. Belykh, V. Belykh, and M. Hasler, "Blinking model and synchronization in small-world networks with a time-varying coupling," Physica D 195, 188-206 (2004).

${ }^{9}$ M. H. V. Belykh and I. Belykh, "Dynamics of stochastically blinking systems. Part I: Finite time propertie,” SIAM J. Appl. Dyn. Syst. 12, 1007-1030 (2013).
${ }^{10}$ J. A. Acebrón, L. L. Bonilla, C. J. P. Vicente, F. Ritort, and R. Spigler, “The Kuramoto model: A simple paradigm for synchronization phenomena," Rev. Mod. Phys. 77, 137-175 (2005).

${ }^{11}$ S. Gupta, A. Campa, and S. Ruffo, "Kuramoto model of synchronization: Equilibrium and nonequilibrium aspects," J. Stat. Mech. Theor. Exp. 8, R08001 (2014).

${ }^{12}$ A. Pikovsky and M. Rosenblum, "Dynamics of globally coupled oscillators: Progress and perspectives," Chaos 25, 097616 (2015).

${ }^{13}$ S. Watanabe and S. H. Strogatz, "Integrability of a globally coupled oscillator array," Phys. Rev. Lett. 70, 2391-2394 (1993).

${ }^{14}$ S. Watanabe and S. H. Strogatz, "Constants of motion for superconducting Josephson arrays," Phys. D Nonlinear Phenom. 74, 197-253 (1994).

${ }^{15} \mathrm{H}$. Tanaka, A. Lichtenberg, and S. Oishi, "First order phase transition resulting from finite inertia in coupled oscillator systems," Phys. Rev. Lett. 78, 2104-2107 (1997).

${ }^{16}$ H. Hong, M. Y. Choi, J. Yi, and K.-S. Soh, "Inertia effects on periodic synchronization in a system of coupled oscillators," Phys. Rev. E 59, 353-363 (1999).

${ }^{17}$ F. Dorfler, M. Chertkov, and F. Bullo, "Synchronization in complex oscillator networks and smart grids," Proc. Natl. Acad. Sci. U.S.A. 110, 2005-2010 (2013).

${ }^{18}$ G. Filatrella, A. H. Nielsen, and N. F. Pedersen, "Analysis of a power grid using a Kuramoto-like model,” Eur. Phys. J. B 61, 485-491 (2008).

19 J. M. V. Grzybowski, E. E. N. Macau, and T. Yoneyama, "On synchronization in power-grids modelled as networks of second-order Kuramoto oscillators," Chaos 26, 113113 (2016)

${ }^{20}$ S.-Y. Ha, Y. Kim, and Z. Li, "Large-time dynamics of Kuramoto oscillators under the effects of inertia and frustration," SIAM J. Appl. Dyn. Syst. 13, 466-492 (2014).

${ }^{21}$ J. Barré and D. Métivier, "Bifurcations and singularities for coupled oscillators with inertia and frustration," Phys. Rev. Lett. 117, 214102 (2016).

${ }^{22}$ I. V. Belykh, B. N. Brister, and V. N. Belykh, "Bistability of patterns of synchrony in Kuramoto oscillators with inertia," Chaos 26, 094822 (2016).

${ }^{23}$ S. Olmi, "Chimera states in coupled Kuramoto oscillators with inertia," Chaos 25, 123125 (2015).

${ }^{24}$ E. A. Martens, S. Thutupalli, A. Fourrière, and O. Hallatschek, "Chimera states in mechanical oscillator networks," Proc. Natl. Acad. Sci. U.S.A. 110, 10563-10567 (2013).

${ }^{25}$ T. Kapitaniak, P. Kuzma, J. Wojewoda, K. Czolczynski, and Y. Maistrenko, "Imperfect chimera states for coupled pendula," Sci. Rep. 4, 6379 (2014).

${ }^{26}$ S. Olmi, A. Navas, S. Boccaletti, and A. Torcini, "Hysteretic transitions in the kuramoto model with inertia," Phys. Rev. E 90, 042905 (2014).

${ }^{27}$ J. A. Acebrón and R. Spigler, "Adaptive frequency model for phase-frequency synchronization in large populations of globally coupled nonlinear oscillators," Phys. Rev. Lett. 81, 2229-2232 (1998).

${ }^{28}$ J. A. Acebrón, L. L. Bonilla, and R. Spigler, "Synchronization in populations of globally coupled oscillators with inertial effects," Phys. Rev. E 62, 3437-3454 (2000).

${ }^{29}$ S. Gupta, A. Campa, and S. Ruffo, "Nonequilibrium first-order phase transition in coupled oscillator systems with inertia and noise," Phys. Rev. E 89, 022123 (2014).

${ }^{30} \mathrm{M}$. Rohden, A. Sorge, M. Timme, and D. Witthaut, "Self-organized synchronization in decentralized power grids," Phys. Rev. Lett. 109, 064101 (2012).

${ }^{31}$ P. J. Menk, J. Heitzig, J. Kurths, and H.-J. Schellnhuber, "How dead ends undermine power grid stability,” Nat. Commun. 5, 3969 (2014).

${ }^{32} \mathrm{P}$. Jaros, Y. Maistrenko, and T. Kapitaniak, "Chimera states on the route from coherence to rotating waves," Phys. Rev. E 91, 022907 (2015).

${ }^{33}$ S. Olmi, E. A. Martens, S. Thutupalli, and A. Torcini, "Intermittent chaotic chimeras for coupled rotators,” Phys. Rev. E 92, 030901 (2015).

${ }^{34}$ G. C. Sethia and A. Sen, "Chimera states: The existence criteria revisited," Phys. Rev. Lett. 112, 144101 (2014).

${ }^{35}$ L. Schmidt, K. Schönleber, K. Krischer, and V. García-Morales, "Coexistence of synchrony and incoherence in oscillatory media under nonlinear global coupling," Chaos 24, 013102 (2014).

${ }^{36}$ A. Yeldesbay, A. Pikovsky, and M. Rosenblum, "Chimeralike states in an ensemble of globally coupled oscillators,” Phys. Rev. Lett. 112, 144103 (2014).

${ }^{37}$ Y.-C. Lai and T. Tel, Transient Chaos. Complex Dynamics on Finite Time Scales (Springer, New York, 2011).

${ }^{38}$ A. Pikovsky and A. Politi, Lyapunov Exponents. A Tool to Explore Complex Dynamics (Cambridge University Press, Cambridge, 2016). 